\title{
Justificación y error en Descartes: un argumento pragmatista en la validación cartesiana del criterio de claridad y distinción
}

\author{
(Justification and error in Descartes: \\ a pragmatist argument in the Cartesian \\ validation of the clearness and \\ distinctness's criterion)
}

Sergio GARCÍA RoDRÍGUEZ

Recibido: 4 de febrero de 2015

Aceptado: 5 de octubre de 2015

\section{Resumen}

La percepción clara y distinta es el elemento sobre el que se asienta la certeza metafísica de Descartes. Con todo, el planteamiento de los argumentos escépticos referidos a la duda metódica cartesiana ha evidenciado la necesidad de hallar una justificación al propio criterio de la percepción clara y distinta. Frente a los intentos basados en la indubitabilidad de la percepción o en la garantía surgida de la bondad divina, se defenderá una justificación alternativa pragmatista.

Palabras clave: Descartes, certeza metafísica, claridad y distinción, justificación, escepticismo, error, pragmatismo

\begin{abstract}
The clear and distinct perception is the element on which Descartes's metaphysical certainty is justified. However, the exposition of the skeptical arguments referred to the Cartesian methodical doubt has shown that is necessary to find another justification for the clearness $\&$ distinctness criterion. From attempts based on indubitability of the perception or warranty arising from the divine goodness, it will be defended a pragmatist alternative justification.
\end{abstract}


Keywords: Descartes, metaphysical certainty, clearness and distinctness, justification, skepticism, error, pragmatism

\section{La certeza metafísica como fundamento seguro del conocimiento}

La cuestión del error encarna para Descartes ${ }^{1}$ uno de los problemas centrales de cuya elucidación depende el resto de su proyecto filosófico. Para comprender dicha importancia es necesario, primeramente, atender al contexto vivido por Descartes, donde el surgimiento de una "nueva ciencia" representada por Galileo, Kepler o Harvey y el resurgir del escepticismo ${ }^{2}$ a consecuencia de la crisis del paradigma filosófico vigente - el aristotélico-escolástico ${ }^{3}$ - marcan a Descartes la necesidad de alcanzar dos objetivos centrales: (1) la obtención de unos fundamentos últimos y seguros que superen los argumentos escépticos; (2) la posibilidad de dar cuenta y continuar con la construcción de esta "nueva ciencia" a partir de tales fundamentos. El error, por tanto, juega un papel indesligable en la cuestión de la búsqueda de los fundamentos últimos, pues los argumentos escépticos se constituirán con vistas a mostrar que no poseemos un criterio por el cual juzgar si en cualquier cuestión a determinar estamos errados o no, de tal manera que podemos equivocarnos incluso en aquellas cuestiones que consideramos más fiables. Así, la obtención de unos fundamentos últimos será sinónimo de superar los argumentos escépticos, es decir, adquirir un conocimiento cierto y seguro en forma de certeza metafisica.

El procedimiento cartesiano para alcanzar las certezas últimas necesita partir del argumento escéptico más radical -encarnado en el "argumento del genio maligno"4-, cuyo zénit hiperbólico hace extensible la posibilidad de error a toda dimen-

\footnotetext{
${ }^{1}$ Las obras de Descartes serán citadas según la edición Adam-Tannery (AT, vol., p.), no obstante, dado que cada uno de los volúmenes incluye más de una obra, se incorporará en la referencia la obra concreta citada.

2 Cf. Popkin, 2011, cap. 2 y 4

${ }^{3}$ La vinculación entre "nueva ciencia" y la crisis de la Escolástica es clara. La "nueva ciencia" se caracteriza por ofrecer nuevas explicaciones -como las nuevas explicaciones sobre el movimiento que se distanciaban totalmente de la explicación aristotélica y que no podian ser asumidas por ésta- con resultados mucho mayores que los que podía ofrecer la ciencia escolástica. El interés por los resultados y su consiguiente búsqueda es algo propio de la nueva ciencia, pues, tal y como Crombie ha señalado, "[...] existe una diferencia básica entre los objetivos de la filosofía de la ciencia medieval y los de toda la filosofía de la ciencia desde Galileo. [...] El principal interés de los científicos desde Galileo ha recaído sobre el siempre creciente ámbito de problemas concretos que la Ciencia puede resolver [...] porque ciertos problemas científicos concretos y específicos pueden ser resueltos satisfactoriamente sólo por una reforma completa de los principios fundamentales. [...] Pero los filósofos medievales de la naturaleza estaban interesados primordialmente [...] por el tipo de saber de la ciencia de la naturaleza: cómo se adecuaba dentro de la estructura general de su metafísica y, si se extendía más, qué relación tenía con la Teología" (Crombie, 1985, p.109).

4 El argumento del genio maligno - tal como Kenny (1968, p.35 y sig.) ha puesto de manifiesto- , a diferencia de otros como el argumento del sueño que solo permite dudar de nuestra corporalidad, posi-
} 
sión epistémica. Desde este punto escéptico de partida, Descartes debe buscar la certeza metafísica descartando para ello "[...] todo aquello en lo cual pueda imaginar la menor duda, lo mismo que si supiera que es por completo falso" 5 . El criterio propuesto por Descartes para superar dicho argumento es el de la "claridad y distinción", de forma que todo aquello que se nos presente bajo dicho carácter poseerá un estatuto indubitable, desvelando así una certeza.

El cogito encarna el ejemplo paradigmático de certeza metafísica, donde, después de rechazar todo aquello dubitable, surge una proposición que supera el argumento escéptico, pues "[...] esta proposición: yo soy, yo existo, es necesariamente verdadera cada vez que la pronuncie, o que la conciba en mi espíritu"6. Así, una vez se alcancen la totalidad de las certezas metafísicas, se construirán deductivamente el resto de conocimientos a partir de ellas, tratando de que cada paso no sea precipitado y que se siga adecuadamente.

La tarea del presente artículo consistirá en analizar de qué formas el criterio de la claridad y la distinción puede justificarse para superar una serie cuestiones vinculadas al error, derivadas de los argumentos escépticos, que imposibilitan la determinación de una percepción clara y distinta real. La elucidación de esta cuestión será aquello que posibilite sostener un fundamento último en forma de certeza metafísica.

\section{El error en la percepción clara y distinta: problemas interpretativos}

El criterio de la claridad y la distinción anteriormente planteado no es suficiente por sí mismo para justificarse, una serie de problemas vinculados al error que recogeré en este apartado evidencian que tal criterio aún debe ofrecer una serie de justificaciones. El problema fundamental del criterio propuesto por Descartes se puede sintetizar en la siguiente pregunta: ¿Cómo sabemos que lo que nosotros percibimos como claro y distinto es realmente claro y distinto? Esta enunciación 7 plan-

bilita una duda radical cuyo alcance propicia el rechazo de todo aquello que se nos presenta como claro y distinto en tanto que podría ser falso, en palabras de Descartes: "tal vez algún dios había podido darme tal naturaleza que siempre me engañase con respecto a las cosas que me parecen más evidentes" (Descartes, Meditaciones metafisicas, AT, IX, p.28). Esta lectura del argumento del genio maligno se enmarca en lo que Murdoch $(1999$, p. 223) ha denominado "interpretación radical". Para una justificación más profunda de esta interpretación del argumento: $C f$. Wilson, 1978, cap. 3.

5 Descartes, Meditaciones metafísicas, AT, IX, pp. 18-19

6 Ibid., p.19

7 Esta cuestión no debe confundirse con el "problema de la memoria" que algunos autores como Williams (1996) han tratado de postular como el problema real de la percepción clara y distinta. El planteamiento sobre el que versará el análisis no se refiere a aquellas percepciones pasadas que yo recuerdo que fueron claras y distintas (y en las que mi memoria podría engañarme), sino a aquellas que en el mismo momento en que las percibo se me presentan con ese carácter, a pesar de que de hecho no lo posean. Sobre esta cuestión, Doney sostiene que las "Present clear and distinct perceptions were 
tea, en suma, el problema de la justificación de las percepciones claras y distintas. Algunos intérpretes como Rubin o Doney 8 han sostenido como criterio justificatorio de las percepciones claras y distintas el carácter indubitable de la intuición con que se nos presentan, pues Descartes mismo parece afirmarlo en determinados fragmentos, como por ejemplo aquel donde se defiende, respecto al cogito, que "[...] no hay duda de que soy, si me engaña; y que me engañe cuanto quiera, él no podrá nunca hacer que yo no sea nada mientras que yo piense ser algo" 9 . Sobre ello, Rubin sostiene que dichas percepciones indubitables poseen además un carácter que nos impele a creerlas -lo que Williams ha denominado "proposiciones irresistibles"poniendo como ejemplo de ello que "[...] the axioms of Euclidean geometry are 'simple and evident' propositions which our understandings compel our wills to affirm" 10 . Aun así, a pesar de que la indubitabilidad conforme un rasgo indicador de tal percepción, ella no será adecuada como criterio justificativo dado que el propio Descartes introduce en las Meditaciones la posibilidad de tener una percepción clara y distinta de proposiciones muy simples -como esos axiomas de la geometría euclidiana a los que se refiere Rubin- y que sin embargo sea falsa:

También dudaremos de todas las otras cosas que nos han parecido muy ciertas en otro momento, incluso de las demostraciones de la matemática y de sus principios, aun cuando sean bastante manifiestos por si mismos, dado que hay hombres que, razonando sobre tales materias, se han equivocado.11

De ese modo, la percepción clara y distinta no obtiene una justificación suficiente apelando únicamente a su propio carácter indubitable, según el propio Descartes ${ }^{12}$, pues ello no garantiza estar a salvo del error. Con todo, Curley ha señalado que el hecho de que pueda haber error no es condición suficiente para poner en duda algo claro y distinto, pues dudar "[...] must always be reasonable doubt in the sense that some valid ground must always be offered for doubting whatever pro-

never subject to doubt" (Doney, 1955, p.325), por lo que parecería que el problema no se sitúa en las percepciones en el momento que las tenemos, sin embargo, Descartes afirma en las Séptimas Respuestas que "[...] hay muy pocos que sepan distinguir entre lo que verdaderamente se percibe $y$ aquello que tan sólo se cree percibir, porque hay muy pocos que tengan el hábito de no servirse más que de percepciones claras y distintas" (AT, VII, p.511). Sería precisamente en esta distinción donde se ubicará el presente artículo, entre tener una percepción clara y distinta y creer que se tiene tal percepción.

8 Cf. Doney, 1955, pp.326 y sig.

9 Descartes, Meditaciones Metafísicas, AT, IX, p.19.

10 Rubin, 1977, p. 198.

11 Descartes, Principios de la filosofia, AT, IX, pp. 26-7.

12 " [...] una percepción clara y distinta de lo que conozco, la cual no bastaría para asegurarme de su verdad si fuese posible que una cosa concebida tan clara y distintamente resultase falsa" Descartes, Meditaciones metafisicas, AT, IX, p.19. 
position is to be doubted"13. Ahora bien, la búsqueda de fundamentos últimos y seguros que caracteriza al proyecto cartesiano implica un proceso de "duda metódica" según el cual hemos de rechazar "[...] todo aquello en lo cual pueda imaginar la menor duda, lo mismo que si supiera que es por completo falso"14. Descartes reconoce que una percepción clara y distinta puede ser errónea, de lo cual se sigue que tenemos motivos para dudar que las percepciones claras y distintas por sí mismas se hayan de aceptar - por ello Descartes trata de hallar una justificación para ellas como se mostrará más adelante-. Una vez evidenciado que el criterio de indubitabilidad es insuficiente para saber si aquello que se presenta como percepción clara y distinta lo es realmente, será necesario dilucidar qué formas de justificación alternativa necesita para confirmarse como tal. En este punto, conviene discernir las causas posibles de error en una percepción clara y distinta con el fin de elucidar qué justificación posibilita validar algo como claro y distinto y, de ese modo, salvar el error. Para tal tarea se requiere una investigación sobre las causas posibles de una percepción clara y distinta errónea, así como una indagación sobre las justificaciones de tal percepción que posibiliten no incurrir en dichos errores. La interpretación sobre las fuentes de error en el criterio de claridad y distinción puede sintetizarse en dos casos:

(a) "Podría ser que erráramos en nuestras percepciones claras y distintas porque nuestra naturaleza estuviera mal constituida, de tal manera que nosotros no pudiéramos discernir cuando nuestras percepciones claras y distintas lo son realmente."

Esta forma de error se derivaría del argumento del genio maligno, permitiendo plantear, no solo una duda en aquello que se nos presenta como evidente, sino una imposibilidad de base en la naturaleza del sujeto para distinguir entre percepciones claras y distintas reales y erróneas, cuya máxima implicación sería el que pudiéramos estar equivocados siempre. De hecho, el propio Descartes recoge esta posibilidad en las Meditaciones:

"Porque puedo persuadirme de que he sido hecho de tal Naturaleza, que me puedo engañar con facilidad aun en las cosas que creo comprender con la mayor evidencia y certeza; teniendo en cuenta, sobre todo, que me acuerdo de haber estimado con frecuencia muchas cosas como verdaderas y ciertas, que luego otras razones me han llevado a juzgar como absolutamente falsas"15

13 Curley, 1978, p.116.

14 Descartes, Meditaciones metafisicas, AT, IX, pp. 18-19.

15 Descartes, Meditaciones metafísicas, AT, IX, p. 55. 
(b) "Es posible que erremos en nuestras percepciones claras y distintas porque hagamos un mal uso de nuestras facultades, de tal manera que achaquemos a algo de forma precipitada el carácter de 'percepción clara y distinta' sin serlo realmente."

Descartes sostiene que el origen del error se halla en un mal uso de las facultades, donde entendimiento y voluntad no se adecúan de forma correcta. El entendimiento es aquella facultad limitada que nos provee de ideas de cosas que es posible afirmar o negar, y, dado que sólo nos provee de tales ideas - no afirma nada sobre ellas-, "[...] se puede decir que no hay en él nunca error alguno"16. La voluntad, sin embargo, es una facultad ilimitada (AT V, p.159; IX, p.40) que, tomando el material presente en el entendimiento, hace juicios, afirma. Será en ese carácter ilimitado de la voluntad donde ésta pueda excederse de tal manera que:

[...] al ser la voluntad mucho más amplia y más extensa que el entendimiento, no la contengo dentro de los límites, sino que la extiendo también a las cosas que no entiendo [...] y escoge el mal por el bien, o lo falso por lo verdadero. Lo que hace que yo me engañe y peque. 17

De esta forma, cuando la voluntad se exceda y sostenga juicios precipitados, es decir, que van más allá de las ideas que ofrece el entendimiento se hará un mal uso de nuestras facultades. Por tanto, el error se producirá al achacar precipitadamente el carácter de "percepción clara y distinta" a algo que no posee tal naturaleza (AT IX, p.43.

Habitualmente dos han sido las formas propuestas para sortear los dos errores anteriores. En el caso de (a), la solución formulada por Descartes fue apelar a la bondad absoluta de Dios. A través de una serie de argumentos ${ }^{18}$, Descartes demuestra la existencia de Dios, cuya perfección comprende la bondad, siendo así incapaz de crearnos con una naturaleza tal que siempre erráramos pues ello implicaría la existencia de malicia en Dios - una imperfección (AT VIII-B, p. 60; VIII, p. 16; IX, pp. 42-3. Por tanto, nuestra naturaleza no estaría mal constituida y el error no puede darse en ella, de tal manera que éste solo se hallaría en la segunda posibilidad. Respecto a (b), Descartes propone el método en el Discurso con vistas a que conduzcamos bien nuestra razón y no hagamos un mal uso de nuestras facultades. Así

\footnotetext{
16 Ibid., p. 45

17 Ibid., p.46

$18 \mathrm{El}$ argumento más conocido es el ontológico, cuya forma es la siguiente:

(1) Poseemos la idea de suprema perfección, la cual posee todas las perfecciones

(2) La existencia necesaria es una perfección .

(3) Aquello que es supremamente perfecto [Dios] existe
} 
pues, si nos adecuamos a las reglas del método, haremos un uso correcto de nuestras facultades y el error no se producirá en nuestras percepciones claras y distintas pues con él, afirma Descartes:

[...] tenía la seguridad de emplear mi razón en todo, si no perfectamente, por lo menos lo mejor que fuera en mi poder. Sin contar con que, aplicándolo, sentía que mi espíritu se iba acostumbrando poco a poco a concebir los objetos con mayor claridad y distinción. 19

Con todo, una serie de escollos lastran estas salidas al problema del error en la percepción clara y distinta. En primer lugar se halla el conocido problema del "círculo cartesiano", ya planteado por Arnauld y Gassendi, donde se señala que el argumento cartesiano incurre en una circularidad justificatoria. Dios, a través de su infinita bondad, es garante de que nuestra naturaleza está bien constituida. Ahora bien, esta justificación exige que previamente sea demostrada la existencia de Dios y dicha demostración - a través de argumentos como el ontológico- debe surgir del sujeto. Por tanto, si el argumento que demuestra la existencia de Dios no puede ser falso porque Dios garantiza que nuestra naturaleza está bien constituida $-\mathrm{y}$ así el argumento-, estaríamos presuponiendo aquello que debemos demostrar. De esta forma, Dios no podría garantizar, antes de la demostración de su existencia, que el argumento ontológico no es erróneo, por lo que Dios no podría validar nuestra naturaleza como bien constituida.

En segundo lugar, el problema a la solución de (b) apunta a que el método por sí mismo es insuficiente como salida, pues recurrir a éste como criterio exclusivo conduce al siguiente problema:

Es posible que sigamos de forma incorrecta el método, incurriendo así en el error de otorgar el carácter de 'percepción clara y distinta' a algo que no posea tal estatuto.

La apelación que hace Descartes al método es, al fin y al cabo, una forma de desplazar un escalón el problema al que debía hacer frente dicha solución. Está claro que el método posee un papel irrenunciable en la propuesta cartesiana, pero el planteamiento del mismo como genuina solución es insuficiente para solventar el problema de la justificación de la percepción clara y distinta, conduciéndonos al error (c). Por tanto, dado que el planteamiento del método es la solución genuina que Descartes defiende ante (b), los casos a tratar serán (a) y (c).

En este punto se observa que las soluciones propuestas por la lectura habitual se ven lastradas por: (1) un problema de circularidad justificatoria -el llamado "círculo cartesiano"-; (2) una insuficiencia del método como forma genuina de solventar el problema del error. En el próximo apartado propondré una salida alternativa que

19 Descartes, Discurso del método, AT, VI, p. 21. 
trate de dar salida a ambos problemas, pues la solución de uno solo de estos problemas supondría aceptar la posibilidad de que aquello que denomináramos «percepción clara y distinta» siguiera sin serlo. Una solución adecuada, por tanto, deberá enfrentarse a ambas cuestiones.

\section{La dimensión pragmatista de Descartes como justificación alternativa}

En el presente apartado se sostendrá la tesis de que existe una "dimensión pragmatista" en la justificación cartesiana de la certeza metafísica, esto es, de lo que es claro y distinto, cuya consideración posibilita el dar una salida alternativa a los problemas del apartado anterior. Para realizar tal tarea se examinará, en primer lugar, el pragmatismo cartesiano con vistas a poder determinar el sentido de la expresión "dimensión pragmatista" y mostrar así, en segundo lugar, la justificación alternativa del criterio de claridad y distinción.

El pragmatismo no es un movimiento filosófico nacido ex novo en el siglo XIX, el propio William James se refirió a éste como "un nuevo nombre para viejas formas de pensar" indicando que "El método pragmático no tiene nada de nuevo. [Ya que] Sócrates fue un adepto a él, y Aristóteles lo usó metódicamente. [Y] Con su ayuda, Locke, Berkeley y Hume hicieron importantes contribuciones"20, siguiendo la misma línea que Dewey, quien consideraba a Francis Bacon como el primer pragmatista. En consecuencia, esta línea interpretativa parece conducirnos a un "pragmatismo avant la lettre", donde defenderé que se enmarca Descartes y cuyo sentido debe ser elucidado con vistas a determinar el alcance de la dimensión pragmatista cartesiana. El pragmatismo avant la lettre, aun cuando sea pragmatista, no debe entenderse como un reflejo exacto del pragmatismo clásico norteamericano, en tanto que, como señala James, "[...] estos antecesores del pragmatismo lo utilizaron parcialmente y sólo fueron sus precursores" 21 , de tal manera que el pragmatismo cartesiano englobará unas determinadas tesis o actitudes que para el pragmatismo clásico son centrales, pero que están lejos de conformar una síntesis completa de la filosofía de James o Dewey. Para concretar dichos rasgos y actitudes conviene establecer un paralelismo con el caso de Francis Bacon, cuya consideración pragmatista justifica Dewey fundamentalmente en el interés por las consecuencias prácticas formulado en la tesis baconiana de que "el conocimiento es poder" - que Dewey denomina "criterio pragmatista" 22 - encaminada hacia el dominio de la naturaleza. Siguiendo esa caracterización baconiana del pragmatismo avant la lettre, entenderé fundamentalmente la "dimensión pragmatista" cartesiana como "[...] una actitud

\footnotetext{
20 James, 2006, pp. 82-3.

21 Ibid., p. 83.

22 Dewey, 1967, p. 29.
} 
para orientarse [...] y de dirigir las miras a lo que sucede más adelante, los frutos, las consecuencias" 23 , pues, siguiendo a Dewey, este es el rasgo más genuino que permite achacar a un autor una consideración pragmatista. Con todo, este interés por los resultados no debe reducirse a un mero consecuencialismo, dado que, según la caracterización pragmatista de Bacon, la obtención de consecuencias útiles en el pragmatismo avant la lettre se vincula a su vez a otras tesis pragmatistas como el rechazo hacia el conocimiento que en última instancia no produce resultados -lo cual se concreta tanto en Bacon como en Descartes en una dura crítica del aristotelismo-, así como una antropología del sujeto activo preocupado por la acción donde el conocimiento está ligado a la intervención -materializándose en ambos autores en la búsqueda del dominio de la naturaleza-, rechazando así la figura de un sujeto puramente contemplativo. De ese modo, a pesar de que el pragmatismo cartesiano carezca de determinados elementos propios del pragmatismo clásico, aquellos elementos que sí se hallan presentes y que son centrales para el pragmatismo posibilitarán la consideración de Descartes como pragmatista, tal y como sucede con Bacon.

Una vez determinado el sentido de la dimensión pragmatista y el modo en que puede considerarse partícipe del pragmatismo, se debe mostrar en qué manera puede ello ser relevante con vistas a la justificación del criterio de claridad y distinción. Respecto al error (a), y asumiendo que el problema del "círculo cartesiano" lastra su solución, es posible obtener una justificación alternativa para defender que nuestra naturaleza no está mal constituida. Descartes, en la proposición 38 de los Principios, explicita un argumento alternativo que ofrece una forma de elucidar si nuestra naturaleza está bien constituida:

[...] no existe defecto en nuestra naturaleza pues es siempre la misma, sean los juicios verdaderos o falsos. ${ }^{24}$

Este argumento alternativo supone, en suma, subsumir (a) en (c), dado que si es posible precisar cuándo nuestros juicios son acertados y cuándo errados, es decir, cuándo seguimos el método de forma adecuada y hacemos un buen uso de nuestras facultades y cuándo no, podremos determinar que nuestra naturaleza no está mal constituida. Partiendo de que el que se dé (a) entraña que nuestra naturaleza esté mal constituida, es decir, que estamos siempre inclinados al error, conseguir un criterio para salvar (c) implicaría que nuestra naturaleza no se inclina siempre al error.

La cuestión a dilucidar en este punto es, entonces, si es posible obtener un criterio por el cual sepamos que hemos seguido el método de forma adecuada y que la percepción obtenida es correcta. Para afrontar tal tarea considero que debemos diri-

\footnotetext{
23 James, 2006, p. 875.

24 Descartes, Principios de la filosofia, AT, IX, p. 41.
} 
girnos a la justificación que Descartes realiza de su método en el Discurso -pues ella ejemplifica aquello que sucede cuando se sigue el método de forma adecuada, ofreciendo así un criterio que nos indica qué sucede cuando seguimos el método de forma adecuada-, donde sostiene que debemos seguir el método que él propone porque "[...] tales frutos he recogido ya de ese método"25. En otras palabras, Descartes propone una justificación del método basada en las "consecuencias útiles" que ha conseguido extraer del método, pues éste es el método que nos permite aprender "[...] a distinguir lo verdadero de lo falso, para ver claro en mis actos y andar seguro por esta vida" 26 en los tres sentidos del árbol de la ciencia-moral, medicina y mecánica-. Por tanto, la validación que Descartes realiza del método atiende a las consecuencias útiles extraídas de él, es decir, una justificación pragmatista del método -el método es válido porque funciona-. La existencia de una dimensión pragmatista del método se ve, asimismo, confirmada por otro tipo de factores como, por ejemplo, la crítica que Descartes realiza al aristotelismo:

No cabe probar de mejor manera la falsedad de los principios de la filosofía de Aristóteles que afirmando que no ha cabido realizar progreso alguno (AT VIII-B, p.26) por medio de ellos después de haber sido respetados durante siglos 27

El rechazo de Descartes a la filosofía aristotélica surge de que, mientras el predominio de ésta durante siglos no ha conllevado progreso alguno, el método que Descartes propone sí ha ofrecido resultados (AT VI, p.62). Por tanto, la validación del método se halla claramente ligada a las consecuencias útiles que éste es capaz de ofrecer, su capacidad para dar frutos -siendo éstos, en el caso del Discurso, los tres tratados sobre dióptrica, meteoros y geometría que habían surgido de la aplicación de su método- y permitir la intervención del sujeto en la naturaleza.

En este punto parece factible ofrecer un "criterio pragmatista" para salvar el error (c): se sabrá que el método se ha seguido de forma adecuada y que su resultado es verdadero porque se ha conseguido extraer frutos de él. En otras palabras, las consecuencias extraídas del método -que funcionan-son aquello que posibilitan saber que no hemos errado y que hemos alcanzado conocimiento. Será precisamente el conocimiento correcto el que nos permitirá " [...] hacernos como dueños y poseedores de la naturaleza" 28 conllevando ello una serie de frutos que conformarán aquello que valide dicho conocimiento.

Una vez se ha solventado el problema central, ello conllevará el que sea posible determinar también que nuestra naturaleza no está mal constituida -que era el otro

\footnotetext{
25 Descartes, Discurso del método, AT, VI, p. 3.

26 Ibid., p. 10.

27 Descartes, Principios de la filosofia, AT, VIII, pp. 18-19.

28 Descartes, Discurso del método, AT, VI, p. 62.
} 
problema a solventar-. En tanto que poseemos un criterio por el cual discernir entre aquello que es erróneo y aquello que no lo es, es posible saber cuándo estamos errados y cuándo no. De esta forma, nuestra naturaleza no está mal constituida porque si así fuera solamente erraríamos, pero, dado que es posible distinguir cuándo erramos y cuando no, es posible, en consecuencia, saber que conocemos la verdad y que nuestra naturaleza no está mal constituida.

En suma, tal y como se ha puesto de manifiesto, Descartes subraya la importancia de las consecuencias prácticas en la justificación del método. Así, la dimensión pragmatista conformará un elemento irrenunciable que ofrezca un argumento alternativo con vistas a validar las propias certezas cartesianas, actuando como criterio, núcleo de una justificación alternativa de las certezas metafísicas. Con todo, esa dimensión pragmatista debe asumirse, no se trata de una simple justificación alternativa que sea posible rechazar en caso de hallar otra posible, sino que defiendo que se trata de un rasgo propio de la filosofía de Descartes, pues ese carácter instrumental ya ha sido señalado por algunos intérpretes 29 . Por último, conviene reiterar que la dimensión a la que he apelado es pragmatista y no meramente consecuencialista, el pragmatismo avant la lettre de Descartes no aspira a representar de forma total el pragmatismo clásico, sino a introducir algunas tesis y actitudes fundamentales para el pragmatismo, como es el interés por las consecuencias prácticas. Si Dewey consideraba a Bacon como "[...] the prophet of a pragmatic conception of knowledge" 30 en base a su máxima "El conocimiento es poder" con la que podíamos dominar la naturaleza, parece factible que Descartes, quien también busca hacernos "dueños y poseedores de la naturaleza", encarne también ese mismo papel.

\section{Conclusión}

Tras analizar la fundamentación del criterio de claridad y distinción, se ha observado que las soluciones propuestas por Descartes según la interpretación habitual no son suficiente pues: (1) validar la constitución de nuestra naturaleza sobre un argumento ontológico implica caer en el problema del "círculo cartesiano"; (2) el argumento mediante el cual el método se convierte en la guía que nos ayuda a conducir de forma adecuada nuestra razón -y así evitar el error-es insuficiente en tanto que debe especificarse algún criterio que nos permita discernir cuándo seguimos de forma adecuada el método para, así, poder saber si estamos errados de hecho o no.

Una vez evidenciados los problemas que plantea la justificación cartesiana clásica de la certeza, el presente artículo ha tratado de mostrar que Descartes sostuvo a

29 Cf. Clarke, 1982, pp. 4-5; Turró, 1989, p. 26.

30 Dewey, 1967, p. 38. 
su vez una forma alternativa de justificar la certeza atendiendo para ello al carácter práctico del conocimiento, esto es, una justificación pragmatista del conocimiento. La tesis que se ha desarrollado defendía que la certeza cartesiana no puede desvincularse de su carácter pragmatista, pues, tanto en su constitución -donde la justificación de ésta apela, en última instancia, a las consecuencias útiles-como en el fin al que ella sirve -movernos de mejor manera por el mundo-, se encuentra vinculada estrechamente a tal dimensión. De manera que una interpretación que trate de eludir esta cuestión deberá enfrentarse a los problemas que se han evidenciado en el apartado 2, y que solo parecen solventarse -y ser además más coherentes con el resto de la propuesta cartesiana- si se considera el papel pragmatista de la certeza.

Sobre esta interpretación pragmatista de Descartes se podría entender que cabe la posibilidad de sustituir la justificación cartesiana de la certeza que apela al argumento ontológico por una justificación puramente pragmatista. Ahora bien, considero que esta lectura, que podría parecer factible en un principio, sería incorrecta, pues, tal y como ha puesto de manifiesto Williams ${ }^{31}$, Descartes no solo dedica gran parte de sus obras a fundamentar la certeza a través de la existencia de Dios (AT, VI, pp.38-9; AT, IX, pp.28-9), sino que una interpretación que negara el peso de tal argumento quedaría invalidada por la consideración que Descartes hace de los ateos. Según ésta, un ateo nunca podría alcanzar la certeza, pues la justificación de las certezas necesita de Dios como garante:

[...] un Ateo, no puede estar cierto de no ser engañado en las cosas que le parecen ser muy evidentes [...] y nunca estará libre del peligro de tenerla, si antes no reconoce un $\operatorname{Dios}^{32}$

Por tanto, a pesar de que el argumento a través de Dios pudiera no justificar la certeza, la interpretación más correcta de Descartes parecería ser aquella que conjugue dos argumentos que validen la certeza -el de la dimensión pragmatista y el basado en la existencia de Dios-. Cabe además recordar en este punto que aunque la validación de nuestra naturaleza como buena se pudiera realizar desde el argumento ontológico porque no existiera circularidad justificatoria, la necesidad de una justificación pragmatista seguiría manifestándose en tanto que, tal y como señalé en el apartado 2, es necesario que se solventen los posibles errores causados por (a) y (c). El error (c), dirigido a la forma correcta de seguir el método, halla su solución únicamente apelando a la dimensión pragmatista como forma de verificar el buen uso del método.

En suma, la justificación pragmatista de Descartes posibilita una validación alternativa de las percepciones claras y distintas, pudiendo, asimismo, dar cuenta

${ }^{31}$ Cf. Williams, 1996, pp. 238-269.

32 Descartes, Meditaciones metafisicas, AT, IX, p.111. 
del «instrumentalismo cartesiano» propio de su pragmatismo avant la lettre que nos presenta a un sujeto preocupado por la acción y las consecuencias útiles, germen de lo que será el pragmatismo clásico.

\section{Referencias bibliográficas}

Clarke, D. (1982): Descartes' philosophy of science, Manchester, Manchester University Press

Crombie, A.C. (1985): Historia de la Ciencia: De San Agustín a Galileo, Madrid, Alianza Ed.

CuRley, E.M. (1978): Descartes against the Skeptics, Oxford, Basil Blackwell

Descartes, R. (1996): Oeuvres de Descartes, edición preparada por Ch. Adam y P. Tannery, París, Vrin, 1897-1913 (12 volúmenes. 11 vols. + vol. 12 bibliografía). Reeditada por Vrin en 1996.

Dewey, J. (1967): Reconstruction in Philosophy, Boston, Beacon Press

Doney, W. (1955): "The Cartesian Circle", Journal of the History of Ideas, Vol. 16, No. 3, pp. 324-38.

James, W. (2006): Pragmatismo, Madrid, Alianza Ed.

Kenny, A. (1968): Descartes. A study of his philosophy, Nueva York, Random House

Murdoch, D. (1999): “The Cartesian Circle”, The Philosophical Review, Vol. 108, No. 2, pp. 221-44.

Popkin, R. (2011): The History of Scepticism from Erasmus to Descartes, Iowa, Grierson Press

RuBIN, R. (1977): "Descartes's Validation of Clear and Distinct Apprehension", The Philosophical Review, Vol. 86, No. 2, (1977), pp. 197-208.

TuRRó, S. (1989): "Estudio introductorio" en El mundo. Tratado de la luz, Barcelona, Anthropos

Williams, B. (1996): Descartes. El proyecto de la investigación pura, Madrid, Ediciones Cátedra.

WiLSON, M. (1978): Descartes, Londres, Routledge and Kegan Paul.

Sergio García Rodríguez

Universidad de las Islas Baleares

grsergio91@hotmail.com 